\title{
Dysfunction of the hypothalamic-pituitary-adrenal axis in male rat offspring with prenatal food restriction: Fetal programming of hypothalamic hyperexcitability and poor hippocampal feedback
}

\author{
YINXIAN WEN ${ }^{1-3}$, SIYUAN CHENG ${ }^{1}$, JUAN LU ${ }^{1}$, XIA HE $^{1}$, ZHEXIAO JIAO $^{1}$, DAN XU ${ }^{1,3}$ and HUI WANG ${ }^{1,3}$ \\ ${ }^{1}$ Department of Pharmacology, Basic Medical School of Wuhan University; ${ }^{2}$ Department of Joint Surgery and \\ Sports Medicine, Zhongnan Hospital of Wuhan University; ${ }^{3}$ Hubei Provincial Key Laboratory of \\ Developmentally Originated Disease, Wuhan, Hubei 430071, P.R. China
}

Received December 3, 2019; Accepted February 22, 2021

DOI: $10.3892 / \mathrm{mmr} .2021 .12537$

\begin{abstract}
Prenatal food restriction (PFR) induces dysfunction of the hypothalamic-pituitary-adrenal (HPA) axis in the adult offspring. The aim of the present study was to identify the underlying mechanism of this process. Pregnant rats were placed on a restricted diet between gestational day 11 and 21 . The offspring were fed with a high-fat diet and were subjected to unpredictable chronic stress (UCS) from postnatal week 17 to 20. A higher serum corticosterone (CORT) level was observed in the PFR fetuses. Although lower arginine vasopressin (AVP), hippocampal vesicular glutamate transporter 2 (vGLUT2) and glutamic acid decarboxylase 65 (GAD65) mRNA expression levels were detected in the hippocampi of PFR fetuses, the ratio of the mRNA expression levels of vGLUT2 and GAD65 was higher compared with that of the controls, which was accompanied by histopathological and ultrastructural abnormalities of both the hypothalamus and hippocampus. However, there were no marked changes in the hippocampal expression levels of glucocorticoids receptor (GR) and mineralocorticoids receptor (MR) or the ratio of MR/GR ratio. After the fetuses had matured, lower serum CORT and adrenocorticotropic hormone (ACTH) levels were observed in PFR rats without UCS when compared with the control. A higher rise rate of serum ACTH was also observed after UCS when compared with that in rats without UCS. Furthermore, the hypothalamic mRNA expression level of corticotrophin-releasing hormone (CRH) was lower in PFR rats without UCS, while expression levels of CRH, AVP, GAD65 and vGLUT2 were enhanced after UCS when compared with
\end{abstract}

Correspondence to: Dr Hui Wang or Dr Dan Xu, Department of Pharmacology, Basic Medical School of Wuhan University, 169 Donghu Road, Wuhan, Hubei 430071, P.R. China

E-mail: wanghui19@whu.edu.cn

E-mail: xuyidan70188@whu.edu.cn

Key words: prenatal food restriction, hypothalamic-pituitary-adrenal axis, intrauterine programming, hypothalamus, hippocampus the control, accompanied by an increased vGLUT2/GAD65 expression ratio. MR mRNA expression was lower, and GR mRNA expression was higher in the hippocampus of the PFR rats without UCS when compared with the control. However, the mRNA expression levels of both MR and GR in the PFR rats were higher compared with those of the control after UCS, which was accompanied histopathological changes in the dentate gyrus, cornu ammonis (CA1) and CA3 areas. In summary, it was suggested that PFR induced fetal alterations of the HPA axis manifesting as hypothalamic hyperexcitability and poor hippocampal feedback, which persisted to adulthood and affected the behavior of the rat offspring.

\section{Introduction}

Intrauterine growth restriction (IUGR), defined as a developing fetus weighing $10 \%$ or two standard deviations less than the mean body weight of normal fetuses of the same gestational age (1), has been proven to be an independent risk factor of adult metabolic diseases, including diabetes mellitus, obesity and coronary heart disease (2). Based on those findings, Barker (2) introduced the theory of the intrauterine programming of adult diseases, namely abnormal changes in the morphology and function of the fetus caused by maternal undernutrition, environmental pollutants and some other adverse factors during pregnancy, which may persist until adulthood and could be the origin of adult diseases (3).

Prenatal food restriction (PFR) causes IUGR of the fetus. It was previously reported that IUGR of the offspring caused by PFR resulted in an abnormal function of the hypothalamic-pituitary-adrenal (HPA) axis (4). The altered intrauterine programming of the HPA axis may be involved in the mechanism underlying the intrauterine origin of adult diseases (4). Poore and Fowden (5) reported that stimulated serum cortisol was detected in pigs with low birth weight in the first 3 months, while an enhanced adrenal responsiveness to insulin-induced hypoglycemia was observed at 12 months of age. Our previous study found that the serum levels of adrenocorticotrophic hormone (ACTH) and corticosterone (CORT) in the adult rat offspring with PFR were lower compared with those in control rats before unpredictable 
chronic stress (UCS), but were significantly increased after the stress, which was accompanied by alterations in HPA axis-associated neuroendocrine metabolism (6). However, the underlying mechanism remains unknown.

Corticotrophin-releasing hormone $(\mathrm{CRH})$ and arginine vasopressin (AVP) are secreted by parvicellular neurons of the paraventricular nucleus (PVN), a composite structure of the hypothalamus, when the body is exposed to a stressor (7). Subsequently, CRH and AVP activate the anterior pituitary, which releases ACTH. ACTH then promotes the release of glucocorticoid (CORT in rodents or cortisol in humans) by the adrenal gland (8). Glucocorticoids activate the glucocorticoid receptor $(\mathrm{GR})$ and mineralocorticoid receptor $(\mathrm{MR})$ in the hippocampus, which then suppress the activity of the PVN in the hypothalamus via a feedback loop (8). As PFR alters the serum CORT levels and the function of the HPA axis in the offspring (6), it was hypothesized that disruption of the modulatory mechanism between the hippocampus and the hypothalamus in the uterus may program the function of the HPA axis to a low basic activity but a high susceptibility in the adult offspring.

In the present study, an IUGR rat model of PFR was constructed, and the male offspring were fed a high-fat diet to magnify the susceptibility of the HPA axis. The adult male rat offspring were then subjected to PFR. Behavioral changes, histopathological changes of the hypothalamus, HPA axis function and the modulatory mechanism between the hippocampus and the hypothalamus were detected, which could help to identify the underlying mechanism of the fetal programming of the HPA axis function due to PFR.

\section{Materials and methods}

Materials. ACTH kits (cat. no. KIP0061) were purchased from the North Institute of Biological Technology (Beijing, China). ELISA kits for rat CORT were obtained from R\&D Systems, Inc. (cat. no. KGE009). TRIzol ${ }^{\circledR}$ reagent was obtained from Thermo Fisher Scientific, Inc. (cat. no. 15596026). The First Strand cDNA Synthesis kit and reverse transcription-quantitative PCR (RT-qPCR) kits were purchased from Takara Biotechnology, Inc. (cat. nos. 6110A and RR039W). Oligonucleotide primers were synthesized by Sangon Biotech Co., Ltd. Isoflurane was purchased from Baxter International, Inc. Other chemicals and agents were of analytical grade.

Animals and treatment. The animal experiments were performed in the Animal Experiment Centre of Wuhan University, and were accredited by the Association for Assessment and Accreditation of Laboratory Animal Care International. All the procedures were in accordance with the National Institutes of Health Guide for the Care and Use of Laboratory Animals (8th edition) (9). All the protocols were approved by Medical Ethics Committee of the Basic Medical School of Wuhan University (approval no. 201719).

A total of 20 female Wistar rats (weight, 180-220 g) and 10 male rats (weight, 260-300 g) were purchased from the Experimental Centre of Hubei Medical Scientific Academy (animal registration no. 2017-0004). All the rats were specific pathogen-free and were aged 3 months. The animal feeding, grouping and treatment were performed as described in our previous study (6). The animals were maintained at a constant temperature $\left(18-22^{\circ} \mathrm{C}\right)$ and humidity (40-60\%), under a controlled 12-h light/dark cycle, with free access to water and standard rodent chow. Two female rats were mated with one male rat overnight after 1 week of acclimation. Mating was confirmed by the appearance of sperm in a vaginal smear the next morning; this day was designated as gestational day (GD) 0 . Pregnant female rats were then transferred to individual cages. Pregnant rats were fed ad libitum or put on a restricted diet $(50 \%$ of the daily food intake of control rats, $\sim 60 \mathrm{~g} / \mathrm{kg}$ body weight) from GD11 until term delivery. The offspring rats were kept in the same condition, but fed a high-fat diet. Rodent chow was purchased from the Experimental Centre of Hubei Medical Scientific Academy. The standard rodent chow contained $21 \% \mathrm{kcal}$ from protein, $68.5 \% \mathrm{kcal}$ from carbohydrate and $10.5 \%$ kcal from fat, while the high-fat diet contained $88.0 \%$ corn flour, $11.5 \%$ lard and $0.5 \%$ cholesterol, which provided $18.9 \% \mathrm{kcal}$ from protein, $61.7 \% \mathrm{kcal}$ from carbohydrate and $19.4 \% \mathrm{kcal}$ from fat. In addition, behavioral changes of the rats were examined using the open-field test, electronic maze experiment and sucrose preference test following the standard protocols $(10,11)$. Animals were anesthetized using $5 \%$ isoflurane at a flow of $0.4 \mathrm{l} / \mathrm{min}$ for the induction of inhalation anesthesia. Then, the animals were sacrificed by decapitation after being anesthetized. The whole brain was dissected for histological examination of the hippocampus and hypothalamus. H\&E staining of the hypothalamus slices was applied to check whether the PVN was included in the hypothalamus samples.

Assay for blood samples. Blood samples $(250 \mu \mathrm{l})$ were collected though the lateral tail vein from the same set of animals before (b)UCS and after (a)UCS. Serum was collected from the whole blood via centrifugation at $1,200 \mathrm{xg}$ and $4{ }^{\circ} \mathrm{C}$ for $10 \mathrm{~min}$. The serum concentrations of ACTH and CORT were determined following our previously reported protocol (6). The serum concentration of ACTH was determined using radioimmunoassay kits, while the concentration of serum CORT was determined with ELISA kits, following the manufacturer's protocol. In addition, the rates of the increase in the serum concentrations of serum ACTH and CORT were calculated as the following formula:

$$
\mathrm{ACTH}(\mathrm{CORT}) \text { Con. Gain rate }(\%)=\frac{\mathrm{ACTH}(\mathrm{CORT}) \text { Con.aucs }-\mathrm{ACTH}(\mathrm{CORT}) \text { con.bucs }}{\mathrm{ACTH}(\mathrm{CORT}) \text { Con.bUCS }} \times 100
$$

$R T$-qPCR assay. Total RNA was collected from $30 \mathrm{mg}$ hippocampal or hypothalamic tissue using TRIzol reagent. Single-strand cDNA was obtained using a First Strand cDNA Synthesis kit. The RT reaction was performed at $50^{\circ} \mathrm{C}$ for $45 \mathrm{~min}$ to reduce the possibility of non-specific amplification products. The primers were designed using Primer Premier 5.0 (PREMIER Biosoft) and the NCBI BLAST database (https://blast.ncbi.nlm.nih.gov/Blast.cgi; National Center for Biotechnology Information) (12). The RT-qPCR assay was performed on a StepOne thermal cycler (Thermo Fisher Scientific, Inc.) using the Takara RT-qPCR kits under the following thermocycling conditions: Initial denaturation at $95^{\circ} \mathrm{C}$ for $30 \mathrm{sec} ; 40$ cycles of denaturation at $95^{\circ} \mathrm{C}$ for $5 \mathrm{sec}$, annealing at $60^{\circ} \mathrm{C}$ for $30 \mathrm{sec}$ and elongation at $72^{\circ} \mathrm{C}$ for $30 \mathrm{sec}$; final extension at $72^{\circ} \mathrm{C}$ for $10 \mathrm{~min}$. Relative standard curves were applied for relative quantification. Details of primers and 
Table I. Primers and conditions for reverse transcription-quantitative PCR.

\begin{tabular}{lllcc}
\hline Genes & \multicolumn{1}{c}{ Forward (5'-3') } & \multicolumn{1}{c}{ Reverse (5'-3') } & Product (bp) & Annealing \\
\hline$\beta$-actin & GTTGCCAATAGTGATGACCT & GGACCTGACAGACTACCTCA & 208 & $54^{\circ} \mathrm{C}, 20 \mathrm{sec}$ \\
CRH & AGAACAACAGTGCGGGCTCA & GCTCCGGTTGCAAGAAATTCA & 196 & $60^{\circ} \mathrm{C}, 30 \mathrm{sec}$ \\
AVP & AAGAGGGCCACATCCGACA & AGGGCAGGTAGTTCTCCTCCTG & 160 & $58^{\circ} \mathrm{C}, 20 \mathrm{sec}$ \\
GAD65 & TGCAGCCTTGGGGATCGGAA & CCCCAAGCAGCATCCACATGCA & 237 & $60^{\circ} \mathrm{C}, 30 \mathrm{sec}$ \\
VGluT2 & TCCACCGGGGTGGCAAAGTT & TGCGATGTATCCGCCCGGAA & 128 & $60^{\circ} \mathrm{C}, 30 \mathrm{sec}$ \\
GR & CACCCATGACCCTGTCAGTC & AAAGCCTCCCTCTGCTAACC & 156 & $61^{\circ} \mathrm{C}, 30 \mathrm{sec}$ \\
MR & TGCATGATCTCGTGAGTGA & AAGTTCTTCCTGGCCGGTAT & 190 & $62^{\circ} \mathrm{C}, 30 \mathrm{sec}$ \\
\hline
\end{tabular}

CRH, corticotrophin-releasing hormone; AVP, arginine vasopressin; GAD65, glutamic acid decarboxylase 65; VGluT2, Vesicular glutamate transporter 2; GR, glucocorticoids receptor; MR, mineralocorticoids receptor.

PCR conditions are listed in Table I. The relative expression of all the target genes, including CRH, AVP, glutamic acid decarboxylase 65 (GAD65), vesicular glutamate transporter 2 (vGLUT2), GR and MR, was standardized against $\beta$-actin expression levels. The Comparative $\mathrm{Cq}$ Method $\left(2^{-\Delta \Delta \mathrm{Cq}}\right.$ Method) was applied to obtain the relative expression level of the target genes (13).

Histological examination. The samples were fixed at $4^{\circ} \mathrm{C}$ in a $4 \%$ paraformaldehyde solution overnight and processed with the paraffin section technique. Sections (thickness, $5 \mu \mathrm{m}$ ) were stained with H\&E using the following protocol: Immersing in xylene for $45 \mathrm{sec}$ and then in propanol for another $45 \mathrm{sec}$, staining with hematoxylin at $30^{\circ} \mathrm{C}$ for $45 \mathrm{sec}$ in a thermostatic bath, rinsing with warm distilled $\mathrm{H}_{2} \mathrm{O}$ for $45 \mathrm{sec}$, staining in eosin at $30^{\circ} \mathrm{C}$ for $30 \mathrm{sec}$ in a thermostatic bath, rinsing with cold distilled $\mathrm{H}_{2} \mathrm{O}$ for $15 \mathrm{sec}$, dipping in propanol for $45 \mathrm{sec}$ and then $45 \mathrm{sec}$ in xylene. Then, the slices were observed under a light microscope at original magnifications of x100 and $\mathrm{x} 400$.

A fetal rat hippocampal tissue sample was randomly selected from each group and fixed at $4^{\circ} \mathrm{C}$ in $2.5 \%$ glutaral solution for $2 \mathrm{~h}$ and at room temperature in $1 \%$ osmium acid solution for $1 \mathrm{~h}$, successively. Then, the samples were dehydrated in increasing ethyl alcohol concentrations (in $50 \%$ ethyl alcohol for $15 \mathrm{~min}, 70 \%$ ethyl alcohol for $15 \mathrm{~min}$, $80 \%$ ethyl alcohol for $15 \mathrm{~min}, 95 \%$ ethyl alcohol for $15 \mathrm{~min}$ and $100 \%$ ethyl alcohol for $15 \mathrm{~min}$ ), embedded in Epon and cut on an Ultrotome $\mathrm{V}$ ultramicrotome into 50-nm sections (LKB). Subsequently, sections were stained with $2 \%(\mathrm{w} / \mathrm{v})$ uranyl acetate and $2 \%$ lead citrate at room temperature for 30 and $5 \mathrm{~min}$, respectively. The changes in ultrastructure were observed and imaged using an H-600 transmission electron microscope (Hitachi, Ltd.) at an original magnification of $\mathrm{x} 15,000$.

Statistical analysis. Data are presented as the mean \pm SEM, from $n \geq 8$ samples/group. All experiments were repeated at least once. A Shapiro-Wilk test was applied to test the normality of the data. Subsequently, unpaired Student's t-test was applied to analyze the continuous data, including the mRNA expression levels and serum concentration of hormones, as well as the ratios of vGLUT1/GAD65 and MR/GR, and the rise rate of serum ACTH, serum CORT and sucrose preference of the rats. Two-way ANOVA and Bonferroni post hoc test were applied to analyze the differences between the control and PFR groups, as well as the differences between with and without UCS groups. Data analysis was performed by using SPSS 17.0 (SPSS, Inc.) and GraphPad Prism 5.0 software (GraphPad Software, Inc.). P $<0.05$ was considered to indicate a statistically significant difference.

\section{Results}

Adult male offspring rats with PFR and high-fat diet $H P A$ axis activity in the rat offspring with maternal PFR before and after PFR. The serum ACTH and CORT concentrations in the adult rat offspring with maternal PFR were lower compared with those in the controls before UCS $(\mathrm{P}<0.01$; Fig. 1A and $\mathrm{C})$. However, no notable differences were observed between the two groups after UCS. In addition, the rate of the increase of serum ACTH in the PFR group was significantly higher compared with that of the control $(\mathrm{P}<0.01$ : Fig. 1B), while the rate of the increase of serum CORT in the PFR group showed no statistically significant changes after UCS (Fig. 1D).

Expression levels of the key regulators of the HPA axis in the hypothalamus and hippocampus of the rat offspring with maternal PFR with or without PFR. vGluT2 and GAD65 are often considered as the markers of the glutamatergic neurons and $\gamma$-aminobutyric acid (GABA)ergic neuron, respectively, as vGluT2 and GAD65 determine the accumulation of glutamic acid (Glu) and GABA at the synapses, respectively, and also are similarly located with the glutamatergic neurons and GABAergic neurons in the central nervous system (14). Therefore, the ratio of the GAD65/vGlut2 was determined to examine the balance of Glu and GABA accumulation at the synapses. CRH mRNA expression level in the hypothalamus was significantly lower in the PFR without UCS group compared with that in controls without UCS ( $\mathrm{P}<0.05$; Fig. 2A), while no significant difference was observed in the AVP, GAD65, and vGLUT2 mRNA expression levels, as well as the vGLUT2/GAD65 ratio (Fig. 2B-E). However, the AVP, GAD65 and vGLUT2 mRNA expression levels, as well as the vGLUT2/GAD65 ratio, were all significantly increased in the 
A

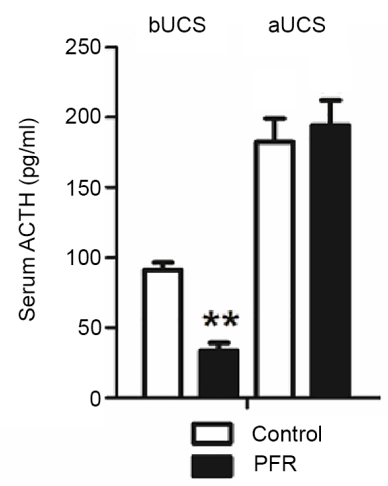

C

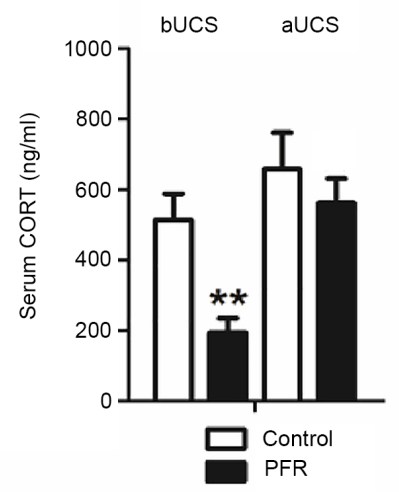

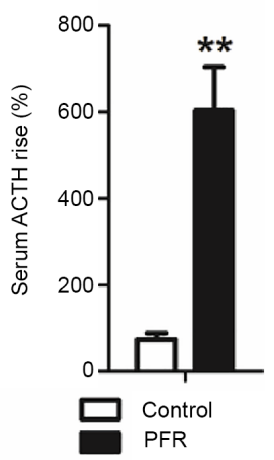

D

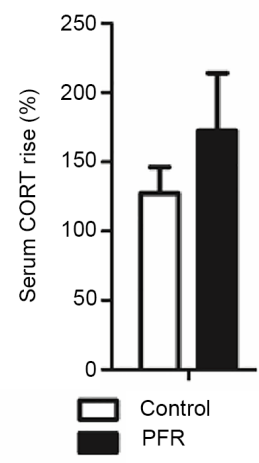

Figure 1. Effects of PFR on the hypothalamic-pituitary-adrenal axis activity in adult male rat offspring. Blood samples were collected from the same set of animals bUCS and aUCS. (A) Serum ACTH concentrations and (B) its increase rate bUCS and aUCS. (C) Serum CORT concentrations and (D) its increase rate. Data are presented as the mean $\pm \mathrm{SEM}, \mathrm{n} \geq 8$. ${ }^{* *} \mathrm{P}<0.01$ vs. control. PFR, prenatal food restriction; ACTH, adrenocorticotropic hormone; CORT, corticosterone; UCS, unpredictable chronic stress; bUCS, before UCS; aUCS, after UCS.

PFR group with UCS when compared with those of the control with UCS ( $\mathrm{P}<0.01$; Fig. 2B-E).

It was found that the MR mRNA expression level in the hippocampus of the rat offspring with maternal PFR was lower compared with the control, while the GR mRNA expression level was higher than the control, when the rats received no UCS ( $\mathrm{P}<0.05$; Fig. 3A and B). However, both the MR and GR mRNA expression levels in the PFR group were upregulated when compared with those of the control, when the rats were exposed to UCS ( $\mathrm{P}<0.05$; Fig. 3A and B). The MR/GR mRNA expression ratio in the PFR group presented a decreasing trend when compared with the control either with or without UCS, while an increasing trend was observed in the ratio with UCS when compared with that without UCS, whereas all these changes showed no statistical difference $(\mathrm{P}<0.05$; Fig. $3 \mathrm{C})$.

With regards to histology, orderly distributed neurons in the dentate gyrus (DG), cornu ammonis (CA) 1 area and CA3 area were observed in the control rats without UCS. However, the neurons in the DG, CA1 and CA3 areas of the rat offspring with PFR were found to be disorderly distributed, with hyperchromatic nuclei in a proportion of the neurons (Fig. 3D). Neurons in the DG, CA1 and CA3 areas were also orderly distributed, with only a few hyperchromatic nuclei observed in the control rats with UCS (Fig. 3D). Moreover, neurons in

the DG, CA1 and CA3 areas of the rat offspring with maternal PFR were further disorderly distributed, with hyperchromatic nuclei observed in several neurons, in the PFR rats with UCS (Fig. 3D). Behavioral changes were also observed in the rat offspring with maternal PFR, and included fewer crossing times and rearing times before UCS and fewer rearing times after the stress in the open field test, as well as decreased total time needed for escape and increased times of correct reaction after UCS (Fig. S1).

\section{Male rat fetuses with maternal PFR}

Ultrastructure and the key regulators of the HPA axis in the hypothalamus and hippocampus. To identify the possible origins of the changes in the adult male rat offspring, the fetuses were further investigated, including the ultrastructural, the histopathological and the expressional changes of the key regulators of the HPA axis in the hypothalamus and hippocampus. Swelling of the mitochondria and endoplasmic reticulum, as well as the cytoplasmic vacuolation, were observed in the neurons located in the hypothalamus of the fetuses with maternal PFR (Fig. 4A). Furthermore, the mRNA expression levels of AVP, GAD65 and vGLUT2 in the hypothalamus were all lower compared with those in the controls, while the vGLUT2/GAD65 ratio was significantly higher in the hypothalamus $(\mathrm{P}<0.05$; Fig. 4B-D).

Changes in the hippocampus were also observed. The serum CORT concentration was significantly higher in the male PFR fetuses compared with that in the controls $(\mathrm{P}<0.05$; Fig. 5A). A notable decreasing trend in the MR expression level was also observed in the male fetuses with maternal PFR (Fig. 5B). However, no statistical significance was observed in the mRNA expression of MR and GR, or the ratio of MR/GR (Fig. 5B and C). Swelling of the mitochondria and endoplasmic reticulum. Swelling of the mitochondria and endoplasmic reticulum, cytoplasmic vacuolation, as well as the aggregation of heterochromatin were observed in the neurons of the hippocampus from the fetuses with maternal PCR, which was rarely observed in the controls (Fig. 5D). Furthermore, the cortex of the hippocampus in the control rats was more mature compared with that in the PFR rats. Neurons in the DG, CA1 and CA3 areas of the control rats were orderly distributed, while neurons in the DG, CA1 and CA3 areas of the rats with maternal PFR were more disordered (Fig. 5E).

\section{Discussion}

Over the past decades, an increasing number of studies have reported that the programmed alteration of the HPA axis serves a key role in the development origin of adult diseases, such as stress and hypertension $(2,15-17)$. Our previous study revealed that the serum levels of ACTH and CORT in adult rat offspring with PFR were lower compared with those in the controls, but were significantly increased after UCS, which was accompanied by alterations in HPA axis-associated neuroendocrinal metabolism (6). Such changes indicated an intrauterine programming of the HPA axis to a low basic activity but a high susceptibility in the adult offspring. Furthermore, a suppressed basic function of the HPA axis in the childhood, but a stimulated sensitivity to stressors during adulthood in the offspring with maternal PFR, were 
A

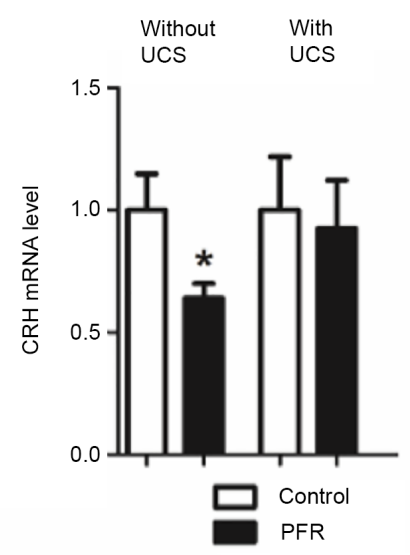

B

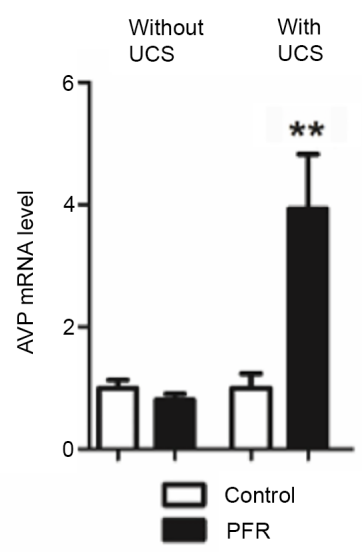

C

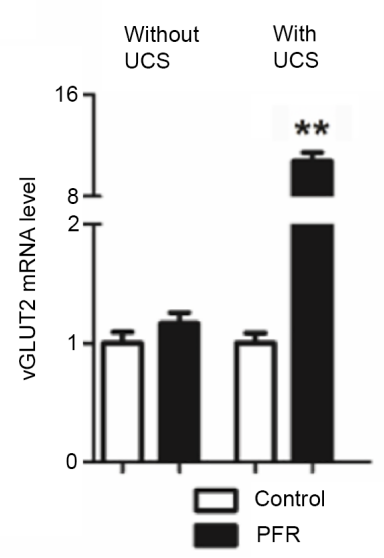

D

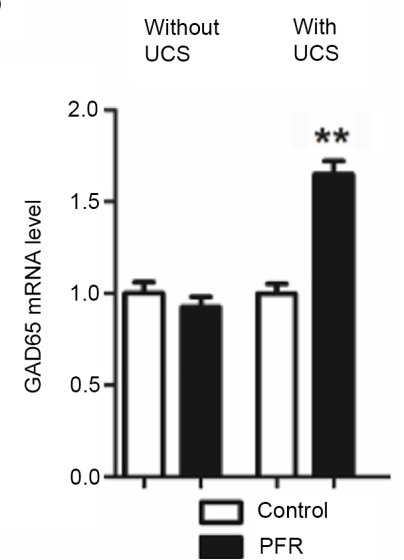

E

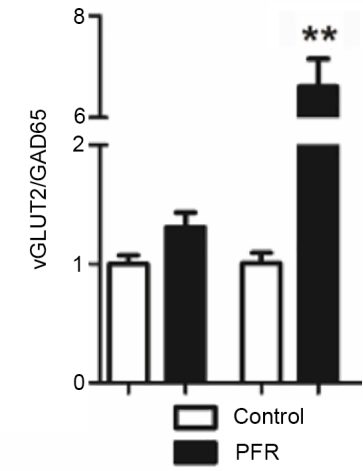

Figure 2. Effects of PFR on the hypothalamic-pituitary-adrenal-axis-related gene expression in the hypothalamus of the adult male rat offspring. Brain samples were collected from the different set of animals with UCS and without UCS. Gene expression levels of (A) CRH and (B) AVP in the hypothalamus of the adult rat offspring bUCS and aUCS. Gene expression levels of (C) vGLUT2 and (D) GAD65, (E) as well as the ratio of the vGLUT2 and GAD65 expression levels in the hypothalamus of the adult rat offspring bUCS and aUCS. Data are presented as the mean $\pm \mathrm{SEM}, \mathrm{n} \geq 8 .{ }^{*} \mathrm{P}<0.05,{ }^{* * *} \mathrm{P}<0.01$ vs. control. PFR, prenatal food restriction; CRH, corticotrophin-releasing hormone; AVP, arginine vasopressin; UCS, unpredictable chronic stress; GAD65, glutamic acid decarboxylase 65; vGLUT2, vesicular glutamate transporter 2; bUCS, before UCS; aUCS, after UCS.

A

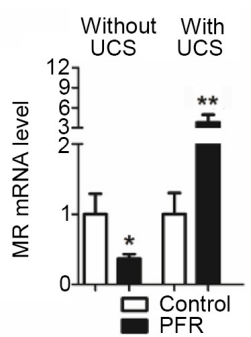

B

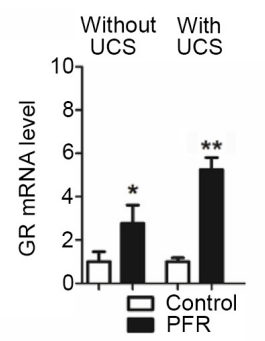

C

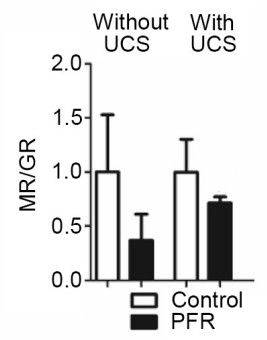

D

Without UCS

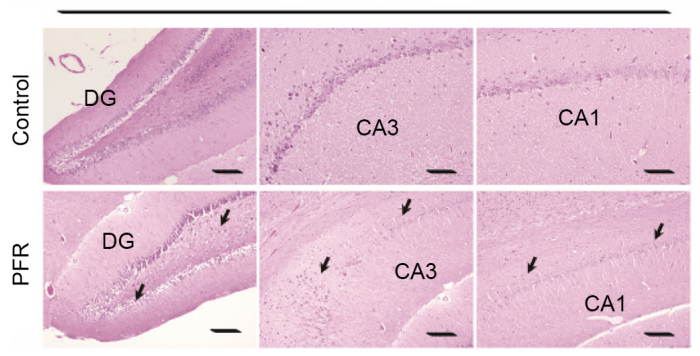

With UCS

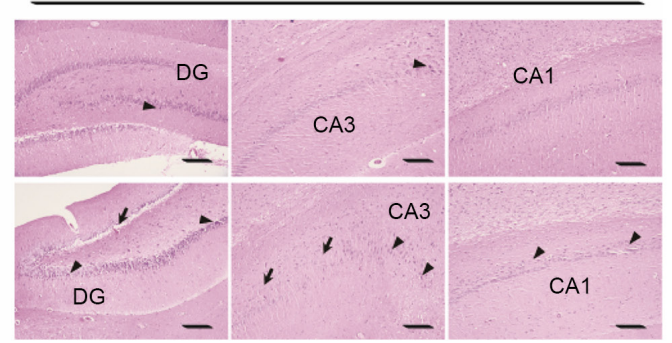

Figure 3. Effects of PFR on the functional gene expression and morphology of the hippocampus in the adult male rat offspring. Brain samples were collected from the different set of animals with UCS and without UCS. Gene expression levels of (A) MR and (B) GR, (C) as well as the ratio of the MR/GR bUCS and aUCS. (D) Histology of the hippocampus was also observed. Arrows indicate disordered neurons, whereas arrowheads indicate hyperchromatic nuclei of the neurons. Data are presented as the mean $\pm \mathrm{SEM}, \mathrm{n}=8$. ${ }^{*} \mathrm{P}<0.05,{ }^{* *} \mathrm{P}<0.01$ vs. control. Scale bar, $100 \mu \mathrm{m}$. DG, dentate gyrus; CA, cornu ammonis; PFR, prenatal food restriction; UCS, unpredictable chronic stress; bUCS, before UCS; aUCS, after UCS. 
A

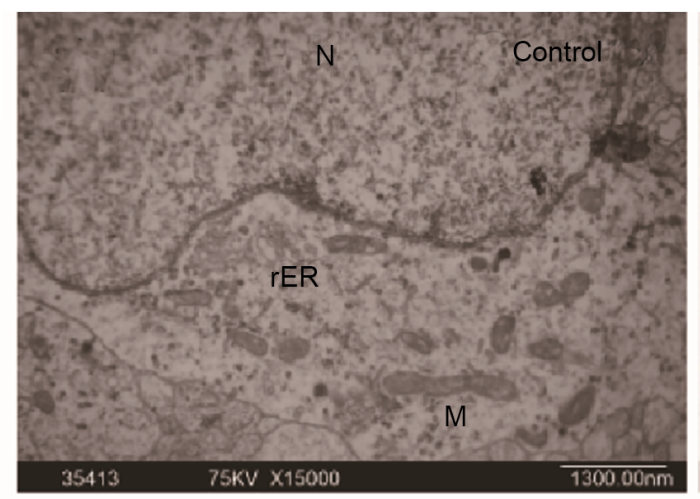

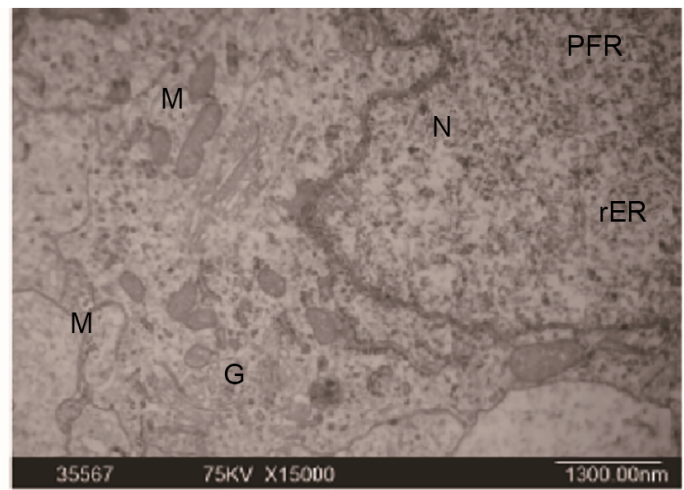

B

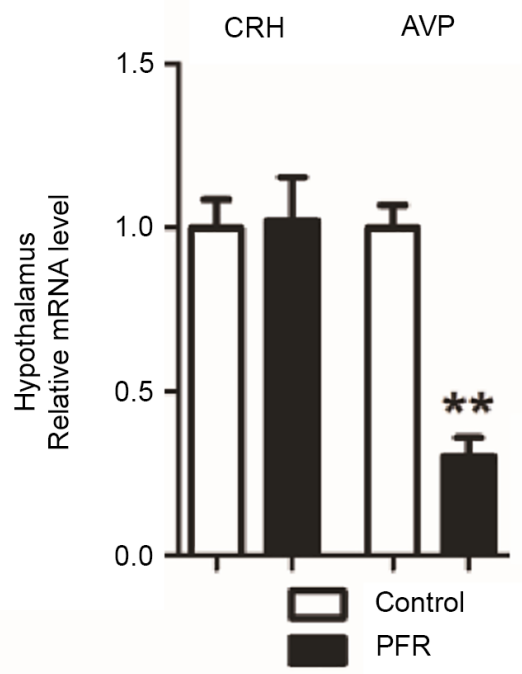

C

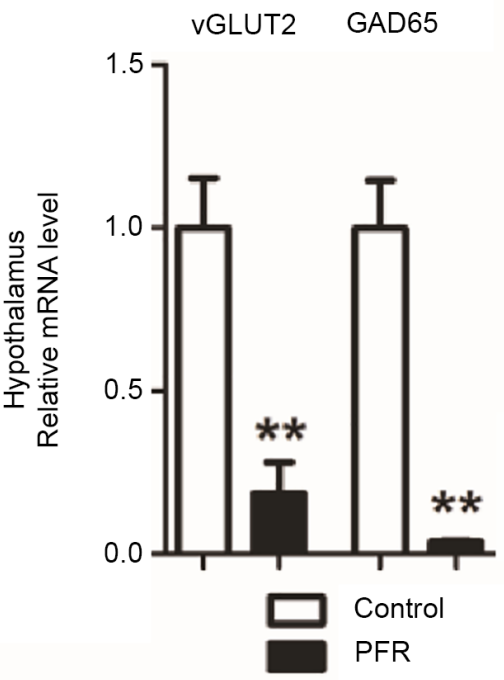

$\mathrm{D}$

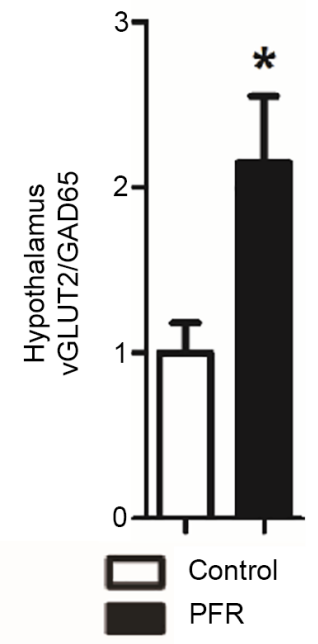

Figure 4. Effects of PFR on the ultrastructure of hypothalamus and hypothalamic-pituitary-adrenal-axis-related gene expression in the hypothalamus of the fetal male rat fetuses. (A) Ultrastructure changes of the fetal hypothalamus. Scale bar, $1.3 \mu$ m. Gene expression levels of (B) CRH and AVP, (C) GAD65 and vGLUT2, (D) as well as the ratio of the vGLUT2/GAD65 expression level in the fetal hypothalamus. Data are presented as the mean $\pm \mathrm{SEM}$, $\geq 8$. * $<0.05$, ${ }^{* *} \mathrm{P}<0.01$ vs. control. N, nucleus; $\mathrm{M}$, mitochondrion; rER, rough surfaced endoplasmic reticulum; G, Golgi apparatus; V, vacuole; PFR, prenatal food restriction; GAD65, glutamic acid decarboxylase 65; vGLUT2, vesicular glutamate transporter 2; CRH, corticotrophin-releasing hormone; AVP, arginine vasopressin.

also reported by other studies (18-21). In the present study, the programmed alteration of HPA axis was also identified in the male adult offspring with maternal PFR. Namely, lower serum concentrations of ACTH and CORT were observed in the PFR rat without UCS, while higher serum concentrations of ACTH and CORT were detected in the PFR rat with UCS, as well as a higher increasing rate of serum ACTH. Moreover, lower expression levels of CRH mRNA in the hypothalamus were found in the PFR rats without UCS, when compared with the controls. However, increases in the serum concentration of ACTH and the mRNA expression level of AVP were detected in the PFR rat with UCS, when compared with the controls. These finding indicated that a low basic activity but a high susceptibility of the HPA axis was induced in the male adult offspring by maternal PFR.

Glu and GABA are the key neurotransmitters in the central nervous system, and the balance of which in the hypothalamus helps to maintain the normal activity of the HPA axis (22). vGLUT2 is the key transporter of Glu at chemical synapses, while GAD65 converts the transformation of Glu to GABA (23). Thus,
vGLUT2 and GAD65 determine the accumulation of Glu and GABA, respectively, at the synapses, and are also synchronically located with glutamatergic neurons and GABAergic neurons in the central nervous system. Furthermore, the gene expression levels of both vGLUT2 and GAD65 are relatively stable in the brain. Thus, vGLUT2 and GAD65 are considered as the markers of the glutamatergic neurons and GABAergic neurons, respectively, and the ratio of vGLUT2/GAD65 is used to determine the balance of Glu and GABA accumulation at the synapses (14). The present study demonstrated that although no significant difference was observed in the vGLUT2 mRNA expression level, the GAD65 mRNA expression level and the expression ratio of vGLUT2/GAD65 in the hypothalamus of the PFR rats without UCS compared with the control, markedly higher vGLUT2 and GAD65 mRNA expression levels, as well as the expression ratio of vGLUT2/GAD65 were observed in the PFR rats with UCS, when compared with the control groups. These findings suggested that a local stimulation of the activity of the hypothalamus may be induced in the male adult offspring by maternal PFR. 
A

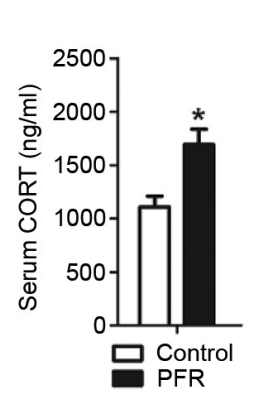

B

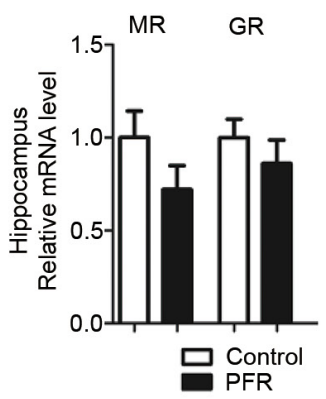

C

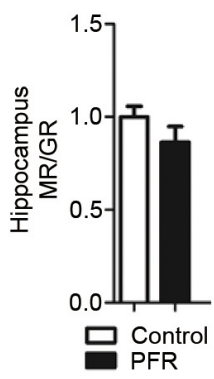

D

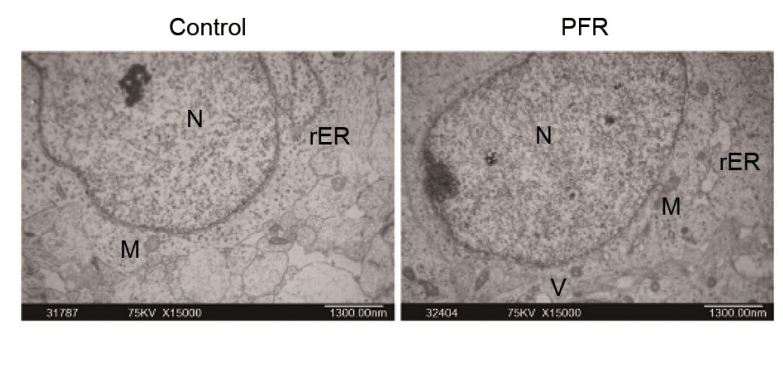

$\mathrm{E}$

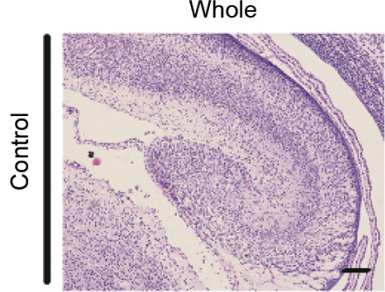

DG

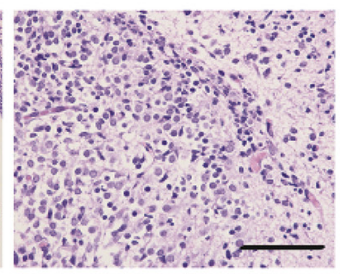

$\mathrm{CA} 3$
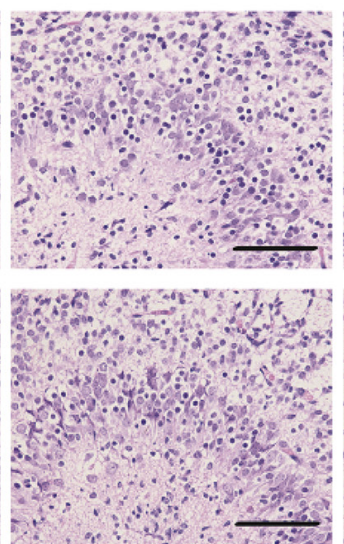

CA1
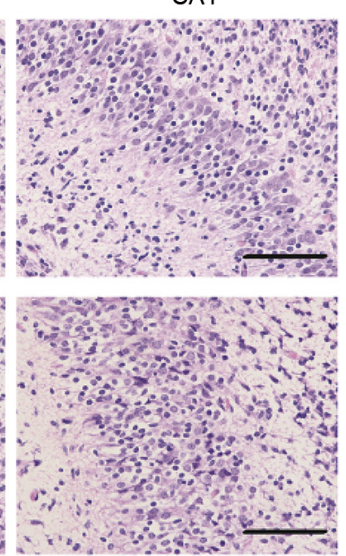

Figure 5. Effects of PFR on the morphology of the hippocampus and the expressions of MR and GR in the hippocampus of the male rat fetuses. (A) Serum CORT concentration, (B) gene expression levels of MR and GR, (C) as well as the ratio of the MR/GR in fetal hippocampus. (D) Ultrastructural changes of the fetal hippocampus. Scale bar, $1.3 \mu \mathrm{m}$. (E) Morphological changes of the hippocampus. Scale bar, $100 \mu \mathrm{m}$. Data are presented as the mean $\pm \mathrm{SEM}, \mathrm{n} \geq 8$. ${ }^{*} \mathrm{P}<0.05$ vs. control. N, nucleus; M, mitochondrion; rER, rough surfaced endoplasmic reticulum; G, Golgi apparatus; V, vacuole; PFR, prenatal food restriction; MR, mineralocorticoid receptor; GR, glucocorticoid receptor; CORT, corticosterone; DG, dentate gyrus; CA, cornu ammonis.

When the body is under stress, the secretion of CRH and AVP from the hypothalamus is stimulated, which then promotes the secretion of ACTH from the anterior pituitary. Subsequently, the ACTH reaches the adrenal gland via the blood circulation, and stimulates the synthesis and secretion of CORT from the adrenal cortex (18). However, when the serum CORT reaches a certain level, the hippocampal GR and MR are activated, participating in the feedback regulation of the HPA axis, which helps to maintain the normal function of the HPA axis (8). As the center regulators of the HPA axis, GR and MR are abundantly expressed in the hippocampus. However, the affinity of MR to CORT is much stronger compared with GR. Thus, almost all CORT binds to MR when the serum CORT level is normal (24). When the serum CORT level increases, particularly under stress, the MR in the hippocampus gets overloaded, and then the GR is activated (25). The activation of GR in the hippocampus induces the secretion of Glu, and successively activates GABAergic neurons via the Glu-GABA interaction, thereby suppressing the function of the hypothalamic $\mathrm{CRH}$ neurons and avoiding the overactivation of the HPA axis (26-28). Thus, the balance of MR and GR in the hippocampus is critical for maintain the function of the HPA axis, which may also determine the local activity of the hypothalamus (29). In addition, the overactivation of GR may induce the influx of $\mathrm{Ca}^{2+}$, which further induces the degeneration and apoptosis of hippocampal neurons, and primarily the cone neurons in the CA3 area and the granular cells in the DG area are the most common victims (30-33).
In the present study, increased GR expression, suppressed MR expression and decreased MR/GR ratio, as well as histopathological changes including decreased number and disordered distribution of neurons in the DG, CA1 and CA3 areas, were observed in the hippocampus of adult male offspring with maternal PFR without UCS. However, immunostaining of the bio-markers of neurons is required to further quantify the cell numbers of the neurons and to evaluate the effect of PFR on the structure of the hippocampus, which is one of the limitations of the current work. The present study demonstrated the mRNA expression levels of both MR and GR were stimulated in the PFR rats with UCS, when compared with the control. Moreover, altered behaviors were observed, including reduced activity in the open field test and poor performance in the maze experiment before and after UCS. All these changes indicated that maternal PFR may programmed a stimulated feedback regulation of hippocampus on the HPA axis at the baseline by activating GR instead of MR in the hypothalamus, which resulted in low basic activity of HPA axis. However, when the animal was subjected to UCS, the programmed feedback regulation from the hippocampus cannot compensate for the overactivation of the HPA axis due to the stimulated local activity of the hypothalamus, leading to the high sensitivity of the HPA axis in the male adult offspring.

Glucocorticoids are key hormones that modulate the growth and maturation of the fetus, and also participate 
in the occurrence of IUGR $(34,35)$. It has been reported that multiple adverse factors during pregnancy, including maternal prenatal food restriction, smoking and alcohol consumption, may induce IUGR, and increased serum glucocorticoids levels are observed in those fetuses with adverse factors during pregnancy (36-40). Our previous studies also revealed that the increased serum glucocorticoid level originated from the maternal blood, which could suppress the development of the HPA axis, thereby causing IUGR in the fetuses $(39,41)$. In the present study, increased serum CORT concentration was observed in the IUGR fetuses induced by PFR, along with decreased AVP, GAD65 and vGLUT2 mRNA expression levels, and an increased vGLUT2/GAD65 ratio. Such changes were similar to the data from the adult male offspring, which indicated that the stimulated potential excitability of the hypothalamus may originate from the fetus. Furthermore, histopathological changes of the hippocampus and hypothalamus were identified in the fetus, which may form the functional changes of the HPA axis. Combined with the evidence from the adult rats, it was suggested that the excessive CORT induced by PFR suppressed the development of both the hippocampus and hypothalamus, but created a stimulated potential excitability of the hypothalamus and a poor feedback regulation from the hippocampus in the fetus, which persisted to the adulthood and manifested as a low basic activity but a high susceptibility in the male adult offspring.

In summary, PFR induced an intrauterine programming of the HPA axis with a low basic activity but a high susceptibility in the male adult offspring under UCS, which appears to have originated from the stimulated potential excitability of the hypothalamus and disturbed feedback regulation from the hippocampus in the fetus.

\section{Acknowledgements}

Not applicable.

\section{Funding}

The present study was supported by the National Natural Science Foundation of China (grant nos. 81430089, 81371483 and 81603214).

\section{Availability of data and materials}

All data generated or analyzed during this study are included in this published article.

\section{Authors' contributions}

Conceptualization: YXW, SYC, DX and HW. Methodology: YXW, SYC, CZ, JL, XH and ZXJ. Software: YXW and SYC. Validation: YXW, SYC, DX and HW. Formal Analysis: YXW, SYC, DX and HW. Investigation: YXW, SYC, CZ, JL, XH and ZXJ. Data curation: YXW and SYC. Original draft preparation: YXW. Review and editing: DX and HW. Visualization: YXW. Supervision: DX and HW. Project administration: YXW, DX and HW. Funding acquisition:
YXW, DX and HW. All authors read and approved the final version of the manuscript.

\section{Ethics approval and consent to participate}

All the protocols were approved by Medical Ethics Committee of the Basic Medical School of Wuhan University (approval no. 201719).

\section{Patient consent for publication}

Not applicable.

\section{Competing interests}

The authors declare that they have no competing interests.

\section{References}

1. Zhang C, Xu D, Luo H, Lu J, Liu L, Ping J and Wang H: Prenatal xenobiotic exposure and intrauterine hypothalamus-pituitary-adrenal axis programming alteration. Toxicology 325: 74-84, 2014.

2. Barker DJ: The developmental origins of chronic adult disease. Acta Paediatr Suppl 93: 26-33, 2004.

3. Clark PM: Programming of the hypothalamo-pituitary-adrenal axis and the fetal origins of adult disease hypothesis. Eur J Pediatr 157 (Suppl 1): S7-S10, 1998.

4. Lesage J, Sebaai N, Leonhardt M, Dutriez-Casteloot I, Breton C, Deloof $S$ and Vieau D: Perinatal maternal undernutrition programs the offspring hypothalamo-pituitary-adrenal (HPA) axis. Stress 9: 183-198, 2006.

5. Poore KR and Fowden AL: The effect of birth weight on hypothalamo-pituitary-adrenal axis function in juvenile and adult pigs. J Physiol 547 (Pt 1): 107-116, 2003.

6. Zhang L, Xu D, Zhang B, Liu Y, Chu F, Guo Y, Gong J, Zheng $X$, Chen $L$ and Wang $H$ : Prenatal food restriction induces a hypothalamic-pituitary-adrenocortical axis-associated neuroendocrine metabolic programmed alteration in adult offspring rats. Arch Med Res 44: 335-345, 2013.

7. Holsboer $\mathrm{F}$ and Ising $\mathrm{M}$ : Stress hormone regulation: Biological role and translation into therapy. Annu Rev Psychol 61: 81-109, 2010.

8. Tasker JG and Herman JP: Mechanisms of rapid glucocorticoid feedback inhibition of the hypothalamic-pituitary-adrenal axis. Stress 14: 398-406, 2011.

9. National Research Council (US); Committee for the Update of the Guide for the Care and Use of Laboratory Animals: Guide for the Care and Use of Laboratory Animals. 8th edition. National Academies Press, Washington, DC, 2011.

10. Brenes Sáenz JC, Villagra OR and Fornaguera Trías J: Factor analysis of forced swimming test, sucrose preference test and open field test on enriched, social and isolated reared rats. Behav Brain Res 169: 57-65, 2006.

11. Deacon RM and Rawlins JN: T-maze alternation in the rodent. Nat Protoc 1: 7-12, 2006.

12. Ye J, McGinnis S and Madden TL: BLAST: Improvements for better sequence analysis. Nucleic Acids Res 34 (Web Server Issue): W6-W9, 2006.

13. Livak KJ and Schmittgen TD: Analysis of relative gene expression data using real-time quantitative PCR and the 2(-Delta Delta C(T)) method. Methods 25: 402-408, 2001.

14. Flak JN, Ostrander MM, Tasker JG and Herman JP: Chronic stress-induced neurotransmitter plasticity in the PVN. J Comp Neurol 517: 156-165, 2009.

15. Smith SM and Vale WW: The role of the hypothalamicpituitary-adrenal axis in neuroendocrine responses to stress. Dialogues Clin Neurosci 8: 383-395, 2006.

16. Sebaai N, Lesage J, Breton C, Vieau D and Deloof S: Perinatal food deprivation induces marked alterations of the hypothalamo-pituitary-adrenal axis in 8-month-old male rats both under basal conditions and after a dehydration period. Neuroendocrinology 79: 163-173, 2004. 
17. Hawkins P, Hanson MA and Matthews SG: Maternal undernutrition in early gestation alters molecular regulation of the hypothalamic-pituitary-adrenal axis in the ovine fetus. J Neuroendocrinology 13: 855-861, 2001.

18. Vieau D, Sebaai N, Leonhardt M, Dutriez-Casteloot I, Molendi-Coste O, Laborie C, Breton C, Deloof S and Lesage J: HPA axis programming by maternal undernutrition in the male rat offspring. Psychoneuroendocrinology 32 (Suppl 1): S16-S20, 2007.

19. Leonhardt M, Lesage J, Croix D, Dutriez-Casteloot I, Beauvillain JC and Dupouy JP: Effects of perinatal maternal food restriction on pituitary-gonadal axis and plasma leptin level in rat pup at birth and weaning and on timing of puberty. Boil Reprod 68: 390-400, 2003.

20. Hawkins P, Steyn C, McGarrigle HH, Saito T, Ozaki T, Stratford LL, Noakes DE and Hanson MA: Effect of maternal nutrient restriction in early gestation on responses of the hypothalamic-pituitary-adrenal axis to acute isocapnic hypoxaemia in late gestation fetal sheep. Exp Physiol 85: $85-96,2000$

21. Tegethoff M, Pryce C and Meinlschmidt G: Effects of intrauterine exposure to synthetic glucocorticoids on fetal, newborn, and infant hypothalamic-pituitary-adrenal axis function in humans: A systematic review. Endocr Rev 30: 753-789, 2009

22. Gohlke JM, Griffith WC and Faustman EM: A systems-based computational model for dose-response comparisons of two mode of action hypotheses for ethanol-induced neurodevelopmental toxicity. Toxicol Sci 86: 470-484, 2005.

23. Herman JP, Mueller NK and Figueiredo H: Role of GABA and glutamate circuitry in hypothalamo-pituitary-adrenocortical stress integration. Ann N Y Acad Sci 1018: 35-45, 2004.

24. de Kloet ER, Meijer OC, de Nicola AF, de Rijk RH and Joëls M: Importance of the brain corticosteroid receptor balance in metaplasticity, cognitive performance and neuro-inflammation. Front Neuroendocrinol 49: 124-145, 2018

25. Kozlovsky N, Matar MA, Kaplan Z, Zohar J and Cohen H: A distinct pattern of intracellular glucocorticoid-related responses is associated with extreme behavioral response to stress in an animal model of post-traumatic stress disorder. Eur Neuropsychopharmacol 19: 759-771, 2009.

26. Treccani G, Musazzi L, Perego C, Milanese M, Nava N, Bonifacino T, Lamanna J, Malgaroli A, Drago F, Racagni G, et al: Stress and corticosterone increase the readily releasable pool of glutamate vesicles in synaptic terminals of prefrontal and frontal cortex. Mol Psychiatry 19: 433-443, 2014.

27. Matthews SG: Early programming of the hypothalamopituitary-adrenal axis. Trends Endocrinol Metab 13: 373-380, 2002.

28. Sapolsky RM and Meaney MJ: Maturation of the adrenocortical stress response: Neuroendocrine control mechanisms and the stress hyporesponsive period. Brain Res 396: 64-76, 1986.

29. Krishnamurthy S, Garabadu D and Joy KP: Risperidone ameliorates post-traumatic stress disorder-like symptoms in modified stress re-stress model. Neuroendocrinology 75: 62-77, 2013.
30. Liao XM, Yang XD, Jia J, Li JT, Xie XM, Su YA, Schmidt MV, Si TM and Wang XD: Blockade of corticotropin-releasing hormone receptor 1 attenuates early-life stress-induced synaptic abnormalities in the neonatal hippocampus. Hippocampus 24: 528-540, 2014.

31. de Quervain DJ, Aerni A, Schelling G and Roozendaal B: Glucocorticoids and the regulation of memory in health and disease. Front Neuroendocrinol 30: 358-370, 2009.

32. Arbel I, Kadar T, Silbermann M and Levy A: The effects of long-term corticosterone administration on hippocampal morphology and cognitive performance of middle-aged rats. Brain Res 657: 227-235, 1994.

33. De Kloet ER, Vreugdenhil E, Oitzl MS and Joëls M: Brain corticosteroid receptor balance in health and disease. Endocr Rev 19: 269-301, 1998.

34. Arends N, Johnston L, Hokken-Koelega A, van Duijn C, de Ridder M, Savage M and Clark A: Polymorphism in the IGF-I gene: Clinical relevance for short children born small for gestational age (SGA). J Clin Endocrinol Metab 87: 2720, 2002.

35. Qiu XS, Huang TT, Deng HY, Shen ZY, Ke ZY, Mei KY and Lai F: Effects of early nutrition intervention on IGF1, IGFBP3, intestinal development, and catch-up growth of intrauterine growth retardation rats. Chin Med Sci J 19: 189-192, 2004.

36. Thamotharan M, Shin BC, Suddirikku DT, Thamotharan S, Garg M and Devaskar SU: GLUT4 expression and subcellular localization in the intrauterine growth-restricted adult rat female offspring. Am J Physiol Endocrinol Metab 288: E935-E947, 2005.

37. Sloboda DM, Moss TJ, Li S, Matthews SG, Challis JR and Newnham JP: Expression of glucocorticoid receptor, mineralocorticoid receptor, and 11beta-hydroxysteroid dehydrogenase 1 and 2 in the fetal and postnatal ovine hippocampus: Ontogeny and effects of prenatal glucocorticoid exposure. J Endocrinol 197: 213-220, 2008.

38. Tan Y, Liu J, Deng Y, Cao H, Xu D, Cu F, Lei Y, Magdalou J, Wu M, Chen L and Wang H: Caffeine-induced fetal rat over-exposure to maternal glucocorticoid and histone methylation of liver IGF-1 might cause skeletal growth retardation. Toxicol Lett 214: 279-287, 2012

39. Xu D, Chen M, Pan XL, Xia LP and Wang H: Dexamethasone induces fetal developmental toxicity through affecting the placental glucocorticoid barrier and depressing fetal adrenal function. Environ Toxicol Pharmacol 32: 356-363, 2011.

40. Chen M, Wang T, Liao ZX, Pan XL, Feng YH and Wang H Nicotine-induced prenatal overexposure to maternal glucocorticoid and intrauterine growth retardation in rat. Exp Toxicol Pathol 59: 245-251, 2007.

41. Xu D, Zhang B, Liang G, Ping J, Kou H, Li X, Xiong J, Hu D, Chen L, Magdalou J and Wang H: Caffeine-induced activated glucocorticoid metabolism in the hippocampus causes hypothalamic-pituitary-adrenal axis inhibition in fetal rats. PLoS One 7: e44497, 2012.

This work is licensed under a Creative Commons Attribution-NonCommercial-NoDerivatives 4.0 International (CC BY-NC-ND 4.0) License. 